\title{
Scanless lattice light sheet microscopy
}

\author{
Bi-Chang Chen ${ }^{1}$ \\ ${ }^{1 .}$ Research Center for Applied Science, Academia Sinica, Taipei, Taiwan
}

Over the last decade, powerful new microscopes have dramatically sharpened biologists' focus on the molecules that animate and propel life [1]. The techniques have improved biologists' ability to visually track the movements of cells' tiniest structures. Noticeably, a new imaging platform, "lattice light sheet microscopy" demonstrated by Chen et al. has shown the ability to collect high-resolution images rapidly and minimizes damage to cells, meaning it can image the three-dimensional activity of molecules, cells, and embryos in fine detail over longer periods [2]. However, in order to construct such a lattice light sheet microscope, a lot of optics components needed to be used since there are at least three 4- $f$ ( $f$ is focal length) system required, which makes optical alignment complicated. Here, we would like to present a simplified lattice light sheet microscopy, which removes two galvanometers in the lattice light sheet microscopy system, meaning that two 4-f systems are exempted to have a lattice light sheet microscopy free from scanning mechanically.

Compared to the lattice light sheet microscopy, in order to have volume scanning via selective plane illumination, the galvanometers serve to translate the light sheet through specimen in $x$ and $z$ [3]. Thus, a pairs of galvanometers with 4- $f$ system is required in between the physical mask and excitation objective. Actually, instead of having galvanometers installed in the system, we could simply shift the projected pattern on the SLM since SLM plane is demagnified onto the sample plane. By this way, the entire microscope becomes very compact and easy for alignment.

In the scanless lattice light sheet microscope system as shown in Figure 1, an expanded laser of $488 \mathrm{~nm}$ laser illuminates a reflective liquid crystal on silicon (LCoS) spatial light modulator (SLM) displaying the calculated phase pattern conjugated to the sample plane and placed before the annular mask as in the lattice light sheet microscopy system. Firstly, a lens (L1) is used in a 2-f configuration to create a diffraction pattern at its focal plane that is the Fourier transform of the incident laser diffracted by the SLM. A custom opaque mask with transmissive annuli is placed at this plane to filter out the unwanted diffraction orders. This desired diffraction pattern then is focused by the excitation objective with the help of a 4- $f$ image setup (L2 and L3) to produce a light sheet and corresponding point spread functions (PSF) at the sample plane. The fluorescence generated within the specimen is collected by an orthogonal detection objective. Both objectives are dipped in a shallow media-filled and temperature controlled bath. A tube lens (L4) images the fluorescence signal filtered by an optical filter onto a camera.

Figure 2 shows the experimental measurements of the lattice light sheet intensity patterns at the sample plane of the excitation objective, which is performed under the scanless lattice light sheet microscope for each Bessel functions at varying distance. These $x z$ excitation intensity profiles are measured by shifting the calculated SLM patterns. The results are in good agreement with theoretically predictions, which means this simplified lattice light sheet microscope is feasible for the bio-imaging applications as lattice light sheet microscopy performs.

Since lattice light sheet microscopy could collect high-resolution images rapidly and minimize damage 
to cells, meaning it can image the three-dimensional activity of molecules, cells, and embryos in fine detail over longer periods than was previously possible, it has drawn a lot of attention in the live image field. To improve the performance of the microscope is required in order to make it more accesses to the public. Here, we demonstrated a simplified lattice light sheet microscope without mechanically scanning, which relaxes the critical optical alignments while performing the construction of lattice light sheet microscope [4].

\section{References:}

[1] D. J. Stephens and V. J. Allan, Science 300 (2003), p. 82-86.

[2] B.-C. Chen, et al. Science 346 (2014), p. 1257998/1-1257998/12.

[3] L. Gao, et al. Nature protocols 9(2014), p.1083-1101.

[4]The control software of the experimental system was licensed from Howard Hughes Medical Institute, Janelia Farm Research Campus

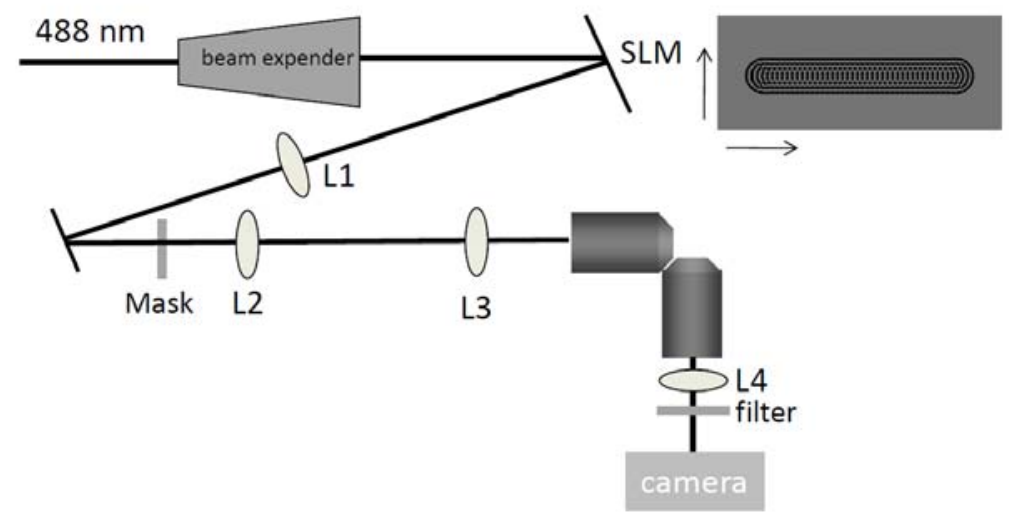

Figure 1. Schematic setup for scanless lattice light sheet microscope : a $488 \mathrm{~nm}$ CW laser source is used for excitation and expanded to cover the desired phase pattern on SLM (HoloEye PLUTO VIS). The diffracting patterns is focused by $\mathrm{L} 1=500 \mathrm{~mm}$ and filtered by mask. The filtered intensity pattern is imaged onto the rear pupil plane of the excitation objective (Nikon, CFI Apo 40XW NIR) through a 4- $f$ system composed of $\mathrm{L} 2=125 \mathrm{~mm}$ and $\mathrm{L3}=250 \mathrm{~mm}$. The emitted fluorescence is collated by the detection objective (Nikon, CFI Apo 40XW NIR) placed orthogonally and imaged onto a sCMOS (Hamamatsu, Orca Flash 4.0) with tube lens L4 = 220 mm.

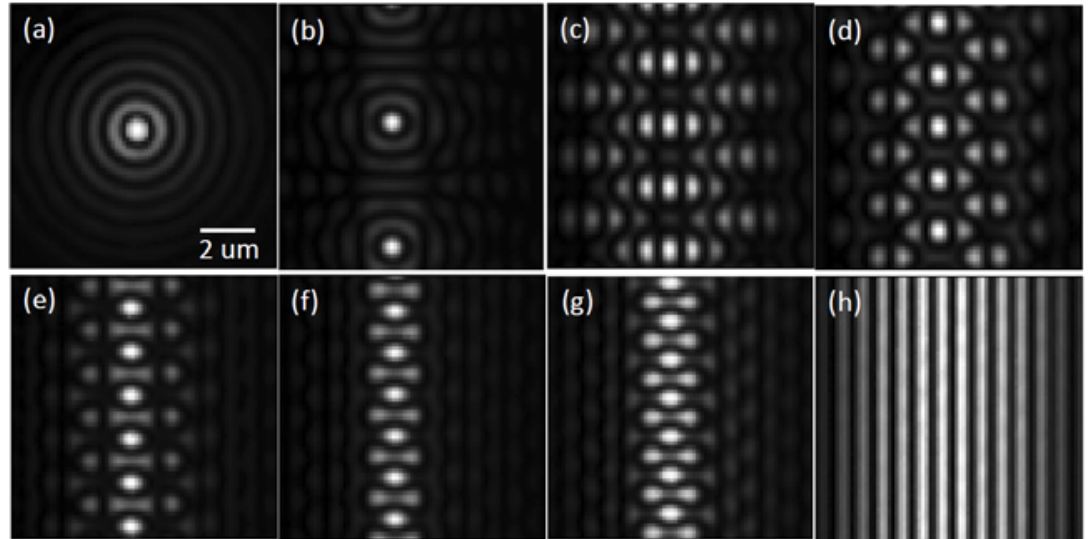

Figure 2. The measured $x z$ cross-sectional intensity profile of the lattice light sheet at the sample: (a) single Bessel beam; (b)-(h), the beam distances between each Bessel beam are 4.80, 2.40, 2.00, 1.64, $1.60,1.48$, and $1.20 \mu \mathrm{m}$, separately. 